\title{
Evaluation of Experimental Hybrids for Powdery Mildew Tolerance in Sunflower (Helianthus annuus L.)
}

\author{
P.G. Suresha ${ }^{1 *}$, Vikas V. Kulkarni ${ }^{2}$, S.M. Supriya ${ }^{1}$, and M.R. Govindappa ${ }^{2}$ \\ ${ }^{1}$ Department of Genetics and Plant Breeding, College of Agriculture, Raichur, Karnataka, India \\ ${ }^{2}$ AICRP on sunflower, MARS, University of Agricultural Sciences, Raichur -584 104, \\ Karnataka, India \\ *Corresponding author
}

A B S T R A C T

Powdery mildew caused by Golovinomyces cichoracearum (DC.) V.P. Heluta (formerly Erysiphe cichoracearum) is a common foliar disease on senescing leaves of cultivated

Keywords

Sunflower, Powdery mildew, Artificial screening.

Article Info

Accepted:

29 June 2017

Available Online:

10 July 2017 sunflower in warmer regions of the world. In India, the powdery mildew disease is becoming major constraint in cultivation of sunflower crop especially during post rainy season. Screening of the sunflower germplasm lines against the disease would be of a great help to identify the resistance source. However, the development of resistant cultivars becomes very important if the crop is to expand into warmer regions where the disease may cause economic losses. Fifty six sunflower hybrids along with one susceptible check Morden were screened under artificial greenhouse conditions during the rabi season of 2013-14 by spraying spore suspension culture at 30 and 45 days after sowing. None of the hybrids screened were immune. However, 31 hybrids were found moderately resistant with less than $25 \%$ PDI while 25 hybrids registered susceptible reaction. The rate of apparent infection ( $r$ ) in hybrids revealed a wide variation among the different hybrids at different intervals. The DMRT (Duncan's multiple Range Test) ranks were assigned to each genotypes based on ' $r$ ' value. The DMRT ranking categorized 57 sunflower genotypes into seven groups.

\section{Introduction}

Sunflower is an important source of nutritious edible oil and considered as good quality oil from health point of view due to presence of polyunsaturated fatty acids with linoleic acid (55-60\%) and oleic acid (25-30\%) which are known to reduce the risk of cardiac related problems. In India, Sunflower is being grown over an area of 0.55 million hectares with an average production and productivity of 0.42 million tonnes and $752 \mathrm{~kg} \mathrm{ha}^{-1}$ respectively during the year 2014-15. Presently Karnataka is the leading state in the country contributing
64.35 and 51.08 per cent to total area and production respectively. It is the second important oilseed crop after groundnut in the state having an area of 0.36 million hectares with production of 0.21 million tonnes. However, productivity (597 $\mathrm{kg} \mathrm{ha}^{-1}$ ) is lesser than the national average of $752 \mathrm{~kg} \mathrm{ha}^{-1}$ (Anon., 2016).

Powdery mildew caused by Golovinomyces cichoracearum (DC.) V.P. Heluta (formerly Erysiphe cichoracearum) is a common foliar 
disease on senescing leaves of cultivated sunflower in warmer regions of the world. In India, the powdery mildew disease in sunflower has become a serious and major constraint in cultivation which was first reported in Bombay (Patel et al., 1949), later in Rajastan (Prasada et al., 1968) and West Bengal (Goswami and Dasgupta, 1981) causing a considerable reduction in yield. The disease originates as minute discoloured speck from which powdery mass radiates in all sides of the leaves. Large area on the aerial parts of the host is covered with white powdery mass containing mycelia and conidia of the fungus (Singh, 1984). Conidia (spores) are primarily dispersed by wind and will germinate on leaves within two to four hours under optimum conditions of high humidity (50-60\%) and temperatures $\left(20-25^{\circ} \mathrm{C}\right)$. A new crop of conidia can be produced within 5 to 7 days leading to rapid spread on the host canopy when conditions are favorable.

Since decade, disease was observed regularly during rabi-summer seasons and under severe conditions, the disease is found to be infecting the cotyledonary leaves up to ray florets. As powdery mildew of sunflower being an obligate pathogen needs a live host to survive. Application of fungicides to manage the disease involves high cost, besides the environmental concern and the insensitivity built up in the pathogen limit their usage (Gullino and Kuijpers 1994). Development of resistant cultivars is the ultimate option to overcome these constraints. The basic step in any successful disease resistance breeding programme is to identify genetic sources through screening a diverse set of germplasm lines for powdery mildew disease. Hence, there is a need for identification of reliable sources for resistance to powdery mildew. So that the resistant genotypes will serve as the potential donors of resistance. Wild Helianthus species represent a valuable reservoir of genes for several biotic stresses which have been successfully introgressed into cultivated sunflower (Seiler, 2008). The development of resistant cultivars will be of great importance to expand sunflower cultivation into warmer regions where the disease may cause economic losses. In this context an attempt was made to screening sunflower germplasm lines against the disease to identify the genetic resistance sources.

\section{Materials and Methods}

\section{Experimental material}

A set of 57 genotypes involving 49 hybrids and 8 checks were sown in pots at MARS, Raichur during the rabi 2013-14 under greenhouse condition. Each hybrid was sown in 2 pots with four seedlings in each pot. The hybrids were screened for reaction to powdery mildew under controlled conditions following artificial inoculation by spraying conidial suspension at 30 and 45 DAS. No crop protection was taken against powdery mildew disease. The disease incidence was recorded from five plants and each plant is divided into bottom, middle, top and observations were recorded as per the disease scale at 45, 60, 75 and 90 days after sowing.

\section{Preparation of inoculum}

The powdery mildew infected leaves were collected from field using camel hair brush. Powdery mass was discharged into 1 per cent sucrose solution and conidial suspension prepared was sprayed on all the entries at 30 and 45 days after sowing.

The genotypes were grouped into different categories using 0-9 disease index scale suggested by Mayee and Datar (1986) for sunflower powdery mildew.

\section{Microscopic observation of pathogen}

For microscopic examination of pathogen, the infected top leaves of medium resistant 
genotypes were scraped gently to dislodge the conidia, then these conidia were stained with lacto phenol blue and observed under motic image capturing microscope at $10 \mathrm{X}$. The number of conidia spores was counted in ten different microscopic fields for four selected medium resistance genotypes, one susceptible genotype and highly susceptible check, Morden. The average number of conidia per microscopic field were analysed using DMRT $(\mathrm{p}=0.05)$.

\section{Statistical analysis}

The powdery mildew disease scored in each hybrid according to 0-9 scale (Table 1) and was converted to per cent disease incidence (PDI) using following formula given by Wheeler (1969). The rate of development of disease (r) at different intervals was also calculated by following formula given by Van der plank (1963).

\section{Results and Discussion}

The full potential of sunflower is far from being exploited due to several abiotic and biotic stresses. The crop suffers from many fungal diseases, among them foliar disease takes a heavy toll by reducing the yield to considerable extent. Among the foliar diseases, powdery mildew caused by Golovinomyces cichoracearum DC is a potential destructive disease in recent years causing severe yield loss. Therefore, resistant breeding appears to be the most important approach in disease management. Availability of resistance source and proper screening procedure is pre-requisite for development of high yielding and powdery mildew resistant hybrids of sunflower.

In the present study fifty six sunflower hybrids along with one susceptible check Morden were evaluated in green house condition under artificial epiphytotic condition. Conidial suspension prepared in one per cent sucrose solution was sprayed on all the entries at 30 and 45 days after sowing. Later the powdery mildew incidence was scored at top, middle and bottom leaves at 15 days interval till plant maturity. Out of 49 experimental hybrids screened under green house condition (Table 2), none were immune or resistant, however 26 hybrids were found to be moderate resistant while 23 hybrids were susceptible. All seven hybrid checks recorded moderate resistance to powdery mildew except KBSH-44 and RSFH-1887. While, the open pollinated variety Morden registered highly susceptible disease reaction (55.35\%). These findings broadly agree with many of earlier reports by pathologists and breeders that no reliable source of resistance was identified (Karunanithi and Dinakaran, 1996). However few reports registered for existence of resistance sources (Hiremat, 1976, Shadakshari et al., 1989, Suresh et al., 1991).

These contradictory findings could be due to differences in scoring methodology, screening procedures and species or race spectrum prevalence. Only small number of accessions from $H$. tuberosus, $H$. nutalii, $H$. maximilliani, $H$. grosseseratus, (Dedic et al., 2012) has been reported to be resistant. However, other researchers found Helianthus nutalii and $H$. grosseseratus to be highly susceptible to powdery mildew in both field and in green house conditions while $H$. rigidus registered moderate resistance to powdery mildew only after inoculation in green house condition (Saliman et al., 1982).

The apparent rate of infection was calculated by using the formula given by Van der plank (1963). This formula is widely used in identification of genotypes with low rate of disease development. The range of ' $r$ ' values among 57 sunflower hybrids ranged from 0.025 to 0.1 indicating the importance of 
infection rate in spreading powdery mildew diseases. The low average ' $r$ ' values indicate less rate of infection compared to higher values.

The average ' $r$ ' values were statistically analysed and compared by using DMRT (0.05). The DMRT ranking categorized 57 sunflower genotypes into seven groups indicating differential rate of infection among different genotypes. Based on apparent rate of infection, hybrids viz., CMS A10 x R2F01120
(B), CMS2A x R-GM-49, CMS A10 x RGM-49, CMS A10 x 83-Br and CMS A10 x R-GM-39 and check hybrids RSFH-1887 and RSFH-10-600 recorded significantly lower ' $r$ ' values indicating the rate of infection in these genotypes is very slow.

Whereas, hybrids CMS 821A x R-GM-41, CMS A10 x R-GM-41 and check hybrid RSFH-130 recorded significantly higher ' $r$ ' values indicating fast spread of disease in these genotypes (Table 3).

Table.1 Disease scoring scale for powdery mildew

\begin{tabular}{|c|c|c|}
\hline Rating & Description & Reaction \\
\hline 0 & No powdery mildew on leaves & Immune \\
\hline 1 & Powdery mildew specks covering $1 \%$ or less area & Highly resistant \\
\hline 3 & Powdery mildew lesions covering $1-10 \%$ of leaf area & Resistant \\
\hline 5 & $\begin{array}{l}\text { Enlarged powdery lesions covering } 11-25 \% \text { of leaf } \\
\text { area }\end{array}$ & $\begin{array}{l}\text { Moderately } \\
\text { resistant/susceptible }\end{array}$ \\
\hline 7 & $\begin{array}{l}\text { Powdery lesions coalesce to form big patches covering } \\
26-50 \% \text { of leaf area }\end{array}$ & Susceptible \\
\hline 9 & $\begin{array}{l}\text { Powdery natches covering } 51 \% \text { or more of leaf area } \\
\text { and defoliation occur }\end{array}$ & Highly susceptible \\
\hline
\end{tabular}

Table.2 Per cent disease severity of powdery mildew disease at 15 days interval in Sunflower hybrids and checks

\begin{tabular}{|c|l|c|c|c|c|c|}
\hline \multirow{2}{*}{ S1.No. } & \multicolumn{2}{|c|}{ Genotypes } & \multicolumn{3}{c|}{ Percent disease severity } & \multirow{2}{*}{ Host } \\
\cline { 3 - 6 } & & $\begin{array}{c}45 \\
\text { DAS }\end{array}$ & $\begin{array}{c}60 \\
\text { DAS }\end{array}$ & $\begin{array}{c}75 \\
\text { DAS }\end{array}$ & $\begin{array}{c}90 \\
\text { DAS }\end{array}$ & reaction \\
\hline 1 & CMS 2A x R-GM-49 & 4.83 & 8.00 & 12.33 & 19.00 & MR \\
\hline 2 & CMS 2A x R-GM-69 & 2.58 & 8.58 & 14.25 & 23.75 & MR \\
\hline 3 & CMS 2A x 83-Br & 4.73 & 8.53 & 13.8 & 23.13 & MR \\
\hline 4 & CMS 2A x R-GM-41 & 3.13 & 5.86 & 14.4 & 25.60 & S \\
\hline 5 & CMS 2A x R-GM-39 & 3.93 & 12.33 & 21.93 & 32.20 & S \\
\hline 6 & CMS 2A x R-393 & 5.93 & 12.53 & 24.53 & 30.40 & S \\
\hline 7 & CMS 2A x R2F01120(B) & 3.66 & 6.86 & 13.4 & 26.26 & S \\
\hline 8 & CMS 821A x R-GM-49 & 2.40 & 6.00 & 14.86 & 26.2 & S \\
\hline 9 & CMS 821A x R-GM-69 & 1.26 & 2.73 & 12.06 & 21.13 & MR \\
\hline 10 & CMS 821A x 83-Br & 0.80 & 1.93 & 15.06 & 27.20 & S \\
\hline 11 & CMS 821A x R-GM-41 & 0.33 & 0.33 & 4 & 21.33 & MR \\
\hline 12 & CMS 821A x R-GM-39 & 1.53 & 3.33 & 14.73 & 24.33 & MR \\
\hline 13 & CMS 821A x R-393 & 2.66 & 3.66 & 6.33 & 20.00 & MR \\
\hline
\end{tabular}




\begin{tabular}{|c|c|c|c|c|c|c|}
\hline 14 & CMS 821A x R2F01120(B) & 0.93 & 1.86 & 10.8 & 19.26 & MR \\
\hline 15 & CMS 850A x R-GM-49 & 1.66 & 4.80 & 14.86 & 27.20 & $S$ \\
\hline 16 & CMS 850A x R-GM-69 & 3.60 & 12.80 & 18.00 & 29.20 & $S$ \\
\hline 17 & CMS 850A x 83-Br & 3.50 & 6.16 & 12.83 & 25.66 & $\mathrm{~S}$ \\
\hline 18 & CMS 850A x R-GM-41 & 0.73 & 3.00 & 8.00 & 21.46 & MR \\
\hline 19 & CMS 850A x R-GM-39 & 0.60 & 2.46 & 9.00 & 24.86 & MR \\
\hline 20 & CMS 850A x R-393 & 0.58 & 1.16 & 8.66 & 23.16 & MR \\
\hline 21 & CMS 850A x R2F01120(B) & 0.83 & 3.16 & 14.33 & 27.00 & $\mathrm{~S}$ \\
\hline 22 & R-10-46-2A x R-GM-49 & 3.80 & 6.20 & 14.40 & 26.93 & $\mathrm{~S}$ \\
\hline 23 & R-10-46-2A x R-GM-69 & 0.73 & 1.86 & 8.80 & 26.2 & $\mathrm{~S}$ \\
\hline 24 & $\mathrm{R}-10-46-2 \mathrm{~A} \times \mathrm{83-Br}$ & 1.58 & 3.66 & 16.5 & 27.5 & $\mathrm{~S}$ \\
\hline 25 & R-10-46-2A x R-GM-41 & 2.11 & 6.33 & 12.5 & 25.44 & $\mathrm{~S}$ \\
\hline 26 & R-10-46-2A x R-GM-39 & 0.6 & 2.06 & 9.73 & 20.13 & MR \\
\hline 27 & R-10-46-2A x R-393 & 1.00 & 3.53 & 9.40 & 18.4 & MR \\
\hline 28 & R-10-46-2A x R2F01120(B) & 1.93 & 3.66 & 9.46 & 18.06 & MR \\
\hline 29 & CMS A4 x R-GM-49 & 1.00 & 2.46 & 11.00 & 23.53 & MR \\
\hline 30 & CMS A4 x R-GM-69 & 1.06 & 2.73 & 10.93 & 25.26 & $\mathrm{~S}$ \\
\hline 31 & CMS A4 x 83-Br & 3.86 & 6.13 & 13.00 & 25.86 & $\mathrm{~S}$ \\
\hline 32 & CMS A4 x R-GM-41 & 1.33 & 2.83 & 12.13 & 27.00 & $\mathrm{~S}$ \\
\hline 33 & CMS A4 x R-GM-39 & 1.66 & 1.73 & 9.73 & 22.6 & MR \\
\hline 34 & CMS A4 x R-393 & 2.30 & 6.80 & 12.50 & 24.30 & MR \\
\hline 35 & CMS A4 x R2F01120(B) & 2.13 & 5.00 & 9.53 & 24.40 & MR \\
\hline 36 & CMS A6 x R-GM-49 & 0.66 & 1.93 & 9.20 & 25.13 & $\mathrm{~S}$ \\
\hline 37 & CMS A6 x R-GM-69 & 0.66 & 2.73 & 10.00 & 18.66 & $\mathrm{MR}$ \\
\hline 38 & CMS A6 x 83-Br & 0.66 & 3.06 & 10.16 & 22.60 & MR \\
\hline 39 & CMS A6 x R-GM-41 & 1.93 & 3.33 & 9.60 & 24.60 & MR \\
\hline 40 & CMS A6 x R-GM-39 & 4.73 & 7.40 & 10.00 & 24.33 & $\mathrm{MR}$ \\
\hline 41 & CMS A6 x R-393 & 1.86 & 4.00 & 16.46 & 27.80 & $\mathrm{~S}$ \\
\hline 42 & CMS A6 x R2F01120(B) & 4.33 & 7.00 & 12.73 & 24.60 & $\mathrm{MR}$ \\
\hline 43 & CMS A10 x R-GM-49 & 6.93 & 10.66 & 20.46 & 28.53 & $S$ \\
\hline 44 & CMS A10 x R-GM-69 & 3.26 & 8.13 & 15.93 & 28.00 & $S$ \\
\hline 45 & CMS A10 x 83-Br & 8.20 & 13.00 & 19.40 & 29.80 & $\mathrm{~S}$ \\
\hline 46 & CMS A10 x R-GM-41 & 0.00 & 0.33 & 1.66 & 22.66 & MR \\
\hline 47 & CMS A10 x R-GM-39 & 6.86 & 8.93 & 16.13 & 26.86 & $\mathrm{~S}$ \\
\hline 48 & CMS A10 x R-393 & 0.86 & 2.46 & 8.73 & 16.73 & MR \\
\hline 49 & CMS A10 x R2F01120(B) & 7.00 & 8.00 & 11.73 & 18.93 & MR \\
\hline 50 & Morden (c) & 2.25 & 17.4 & 26.35 & 55.35 & HS \\
\hline 51 & RSFH-130 (c) & 0.26 & 1.46 & 5.40 & 18.86 & $\mathrm{MR}$ \\
\hline 52 & KBSH-44 (c) & 2.36 & 6.90 & 15.20 & 25.50 & $\mathrm{~S}$ \\
\hline 53 & KBSH-53 (c) & 1.46 & 3.80 & 5.20 & 20.00 & $\mathrm{MR}$ \\
\hline 54 & GK-202 (c) & 1.66 & 2.53 & 7.86 & 21.00 & MR \\
\hline 55 & SB-207 (c) & 0.93 & 2.53 & 5.86 & 19.93 & $\mathrm{MR}$ \\
\hline 56 & RSFH1887 (c) & 7.53 & 11.33 & 15.66 & 26.26 & $\mathrm{~S}$ \\
\hline 57 & RSFH-10-600 (c) & 5.60 & 8.33 & 10.93 & 21.06 & $\mathrm{MR}$ \\
\hline
\end{tabular}

Note: MR: moderate resistant; S: susceptible; HS: highly susceptible 
Table.3 Apparent rate of infection 'r' of Powdery mildew at different stages of Crop growth in sunflower

\begin{tabular}{|c|c|c|c|c|c|c|}
\hline \multirow[b]{2}{*}{$\begin{array}{l}\text { Sl. } \\
\text { No. }\end{array}$} & \multirow[b]{2}{*}{ Crosses } & \multicolumn{3}{|c|}{ Rate of spread 'r' at } & \multirow[b]{2}{*}{$\begin{array}{c}\text { Average } \\
\text { 'r' }\end{array}$} & \multirow[b]{2}{*}{$\begin{array}{c}\text { DMRT } \\
\text { Ranks }\end{array}$} \\
\hline & & $\begin{array}{c}45-60 \\
\text { DAS }\end{array}$ & $\begin{array}{c}60-75 \\
\text { DAS }\end{array}$ & $\begin{array}{l}75-90 \\
\text { DAS }\end{array}$ & & \\
\hline 1 & CMS 2A x R-GM-49 & 0.036 & 0.032 & 0.034 & 0.034 & $\mathrm{CD}$ \\
\hline 2 & CMS 2A x R-GM-69 & 0.084 & 0.038 & 0.042 & 0.055 & $\mathrm{ABCD}$ \\
\hline 3 & CMS 2A x 83-Br & 0.042 & 0.036 & 0.042 & 0.040 & $\mathrm{BCD}$ \\
\hline 4 & CMS 2A x R-GM-41 & 0.044 & 0.066 & 0.048 & 0.053 & $\mathrm{ABCD}$ \\
\hline 5 & CMS 2A x R-GM-39 & 0.082 & 0.046 & 0.035 & 0.054 & $\mathrm{ABCD}$ \\
\hline 6 & CMS 2A x R-393 & 0.055 & 0.055 & 0.020 & 0.043 & $\mathrm{ABCD}$ \\
\hline 7 & CMS 2A x R2F01120(B) & 0.044 & 0.049 & 0.056 & 0.050 & $\mathrm{ABCD}$ \\
\hline 8 & CMS 821A x R-GM-49 & 0.064 & 0.067 & 0.047 & 0.059 & $\mathrm{ABCD}$ \\
\hline 9 & CMS 821A x R-GM-69 & 0.052 & 0.106 & 0.045 & 0.068 & $\mathrm{ABCD}$ \\
\hline 10 & CMS 821A x 83-Br & 0.059 & 0.146 & 0.050 & 0.085 & $\mathrm{ABC}$ \\
\hline 11 & CMS 821A x R-GM-41 & 0.000 & 0.169 & 0.125 & 0.098 & $\mathrm{AB}$ \\
\hline 12 & CMS 821A x R-GM-39 & 0.053 & 0.107 & 0.041 & 0.067 & $\mathrm{ABCD}$ \\
\hline 13 & CMS 821A x R-393 & 0.022 & 0.038 & 0.087 & 0.049 & $\mathrm{ABCD}$ \\
\hline 14 & CMS 821A x R2F01120(B) & 0.047 & 0.123 & 0.045 & 0.072 & $\mathrm{ABCD}$ \\
\hline 15 & CMS 850A x R-GM-49 & 0.073 & 0.083 & 0.051 & 0.069 & $\mathrm{ABCD}$ \\
\hline 16 & CMS 850A x R-GM-69 & 0.091 & 0.027 & 0.042 & 0.053 & $\mathrm{ABCD}$ \\
\hline 17 & CMS 850A x 83-Br & 0.039 & 0.054 & 0.057 & 0.050 & $\mathrm{ABCD}$ \\
\hline 18 & CMS 850A x R-GM-41 & 0.096 & 0.069 & 0.076 & 0.080 & $\mathrm{ABCD}$ \\
\hline 19 & CMS 850A x R-GM-39 & 0.095 & 0.091 & 0.080 & 0.089 & $\mathrm{ABC}$ \\
\hline 20 & CMS 850A x R-393 & 0.047 & 0.139 & 0.077 & 0.088 & $\mathrm{ABC}$ \\
\hline 21 & CMS 850A x R2F01120(B) & 0.091 & 0.109 & 0.053 & 0.084 & $\mathrm{ABCD}$ \\
\hline 22 & R-10-46-2A x R-GM-49 & 0.034 & 0.062 & 0.052 & 0.050 & $\mathrm{ABCD}$ \\
\hline 23 & R-10-46-2A x R-GM-69 & 0.063 & 0.108 & 0.087 & 0.086 & $\mathrm{ABC}$ \\
\hline 24 & $\mathrm{R}-10-46-2 \mathrm{~A} \times \mathrm{83}-\mathrm{Br}$ & 0.057 & 0.110 & 0.043 & 0.070 & $\mathrm{ABCD}$ \\
\hline 25 & R-10-46-2A x R-GM-41 & 0.076 & 0.050 & 0.058 & 0.061 & $\mathrm{ABCD}$ \\
\hline 26 & R-10-46-2A x R-GM-39 & 0.083 & 0.109 & 0.057 & 0.083 & $\mathrm{ABCD}$ \\
\hline 27 & R-10-46-2A x R-393 & 0.086 & 0.069 & 0.052 & 0.069 & $\mathrm{ABCD}$ \\
\hline
\end{tabular}




\begin{tabular}{|c|l|c|c|c|c|c|}
\hline 28 & R-10-46-2A x R2F01120(B) & 0.044 & 0.067 & 0.050 & 0.054 & ABCD \\
\hline 29 & CMS A4 x R-GM-49 & 0.061 & 0.106 & 0.061 & 0.076 & ABCD \\
\hline 30 & CMS A4 x R-GM-69 & 0.064 & 0.098 & 0.067 & 0.077 & ABCD \\
\hline 31 & CMS A4 x 83-Br & 0.032 & 0.055 & 0.056 & 0.048 & ABCD \\
\hline 32 & CMS A4 x R-GM-41 & 0.051 & 0.104 & 0.066 & 0.074 & ABCD \\
\hline 33 & CMS A4 x R-GM-39 & 0.003 & 0.121 & 0.066 & 0.063 & ABCD \\
\hline 34 & CMS A4 x R-393 & 0.075 & 0.044 & 0.053 & 0.057 & ABCD \\
\hline 35 & CMS A4 x R2F01120(B) & 0.059 & 0.046 & 0.075 & 0.060 & ABCD \\
\hline 36 & CMS A6 x R-GM-49 & 0.072 & 0.109 & 0.080 & 0.087 & ABC \\
\hline 37 & CMS A6 x R-GM-69 & 0.096 & 0.092 & 0.048 & 0.079 & ABCD \\
\hline 38 & CMS A6 x 83-Br & 0.104 & 0.085 & 0.063 & 0.084 & ABCD \\
\hline 39 & CMS A6 x R-GM-41 & 0.037 & 0.075 & 0.075 & 0.062 & ABCD \\
\hline 40 & CMS A6 x R-GM-39 & 0.032 & 0.022 & 0.071 & 0.041 & ABCD \\
\hline 41 & CMS A6 x R-393 & 0.052 & 0.103 & 0.045 & 0.067 & ABCD \\
\hline 42 & CMS A6 x R2F01120(B) & 0.034 & 0.044 & 0.054 & 0.044 & ABCD \\
\hline 43 & CMS A10 x R-GM-49 & 0.031 & 0.051 & 0.029 & 0.037 & CD \\
\hline 44 & CMS A10 x R-GM-69 & 0.064 & 0.051 & 0.048 & 0.054 & ABCD \\
\hline 45 & CMS A10 x 83-Br & 0.034 & 0.032 & 0.038 & 0.035 & CD \\
\hline 46 & CMS A10 x R-GM-41 & 0.000 & 0.108 & 0.190 & 0.100 & A \\
\hline 47 & CMS A10 x R-GM-39 & 0.019 & 0.045 & 0.043 & 0.036 & CD \\
\hline 48 & CMS A10 x R-393 & 0.071 & 0.089 & 0.049 & 0.070 & ABCD \\
\hline 49 & CMS A10 x R2F01120(B) & 0.010 & 0.028 & 0.038 & 0.025 & D \\
\hline 50 & Morden & 0.147 & 0.035 & 0.082 & 0.088 & ABC \\
\hline 51 & RSFH-130 & 0.116 & 0.090 & 0.094 & 0.100 & A \\
\hline 52 & KBSH-44 & 0.074 & 0.058 & 0.043 & 0.058 & ABCD \\
\hline 53 & KBSH-53 & 0.065 & 0.022 & 0.101 & 0.063 & ABCD \\
\hline 54 & GK-202 & 0.029 & 0.079 & 0.076 & 0.061 & ABCD \\
\hline 55 & SB-207 & 0.068 & 0.058 & 0.092 & 0.073 & ABCD \\
\hline 56 & RSFH1887 & 0.020 & 0.052 & 0.033 & CD \\
\hline 57 & RSFH-10-600 & 0.025 & 0.043 & 0.033 & CD \\
\hline
\end{tabular}


Int.J.Curr.Microbiol.App.Sci (2017) 6(7): 2716-2728

Table.4 Golovinomyces cichoracearum conidial population on selected promising moderate resistant sunflower genotypes

\begin{tabular}{|c|c|c|c|c|c|c|c|c|c|c|c|c|c|c|}
\hline \multirow{2}{*}{ Genotypes } & \multirow{2}{*}{ PDS } & \multirow{2}{*}{$\begin{array}{l}\text { Host } \\
\text { reaction }\end{array}$} & \multicolumn{10}{|c|}{ No. of conidia spore/microscopic field } & \multirow{2}{*}{ Mean } & \multirow{2}{*}{$\begin{array}{l}\text { DMRT } \\
\text { Ranks }\end{array}$} \\
\hline & & & 1 & 2 & 3 & 4 & 5 & 6 & 7 & 8 & 9 & 10 & & \\
\hline $\mathrm{R}-10-46-2 \mathrm{~A} \times \mathrm{R}-393$ & 18.40 & MR & 21 & 32 & 39 & 46 & 17 & 49 & 53 & 58 & 17 & 14 & 34.6 & $\mathrm{E}$ \\
\hline $\begin{array}{l}\text { R-10-46-2A x } \\
\text { R2F01120(B) }\end{array}$ & 18.06 & MR & 16 & 24 & 19 & 32 & 38 & 29 & 37 & 24 & 32 & 23 & 27.4 & $\mathrm{E}$ \\
\hline CMS A10 x R-393 & 16.73 & MR & 200 & 180 & 160 & 150 & 140 & 130 & 140 & 155 & 145 & 125 & 152.5 & $\mathrm{C}$ \\
\hline CMS A10 x R2F01120(B) & 18.93 & MR & 64 & 102 & 117 & 103 & 109 & 74 & 132 & 114 & 126 & 88 & 102.9 & $\mathrm{D}$ \\
\hline CMS 850A x R-GM-69 & 29.20 & $S$ & 215 & 220 & 210 & 180 & 160 & 150 & 180 & 205 & 225 & 190 & 193.5 & B \\
\hline MORDEN & 55.35 & HS & 380 & 350 & 410 & 390 & 340 & 320 & 400 & 450 & 390 & 430 & 386.0 & A \\
\hline
\end{tabular}


Fig.1 Microphotograph showing conidia and conidiophores of G. cichoracearum at 45X
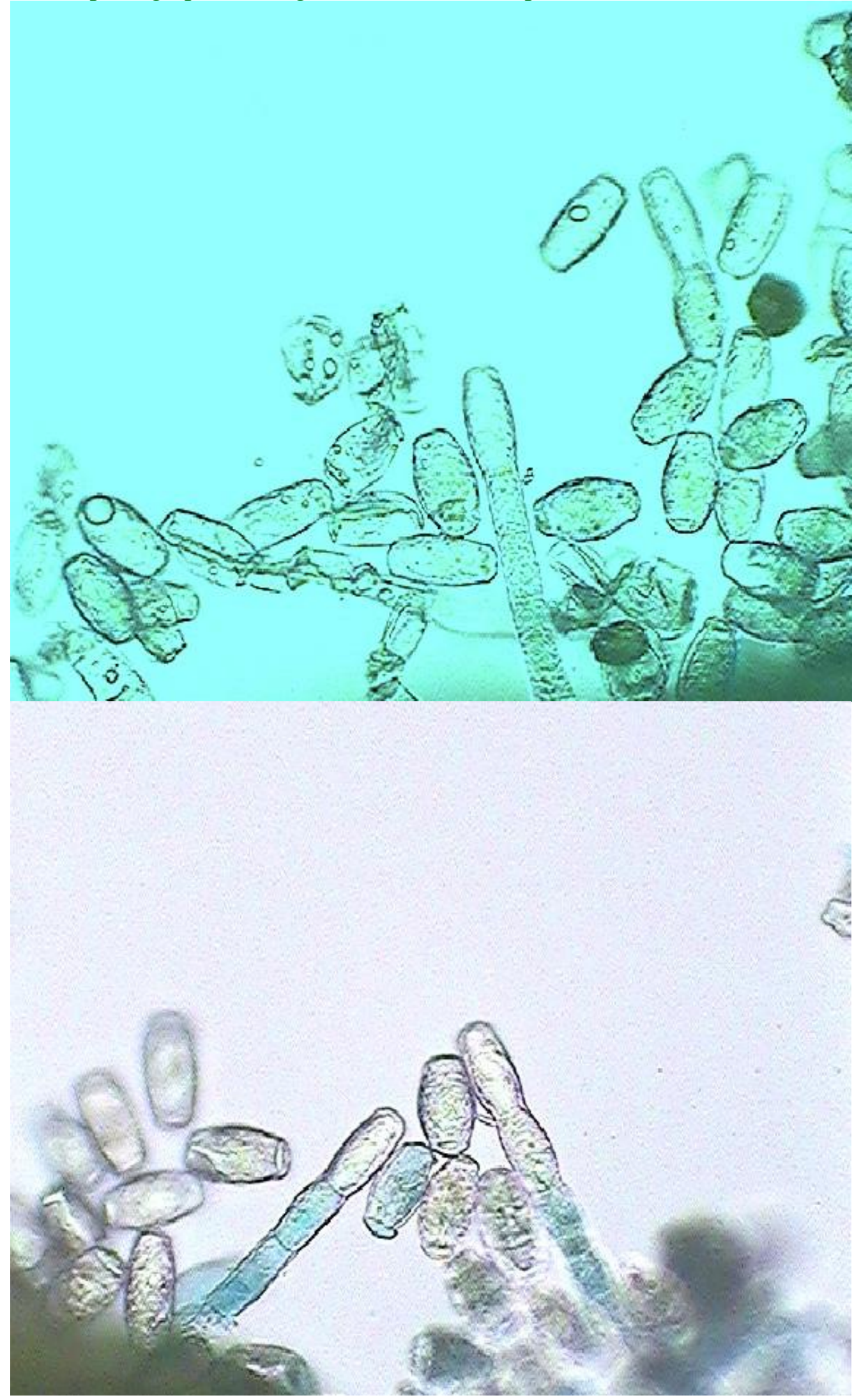
Fig.2 Microphotograph showing number of conidia spore per microscopic field of $G$. cichoracearum in moderate resistant hybrids and susceptible check Morden at 10X

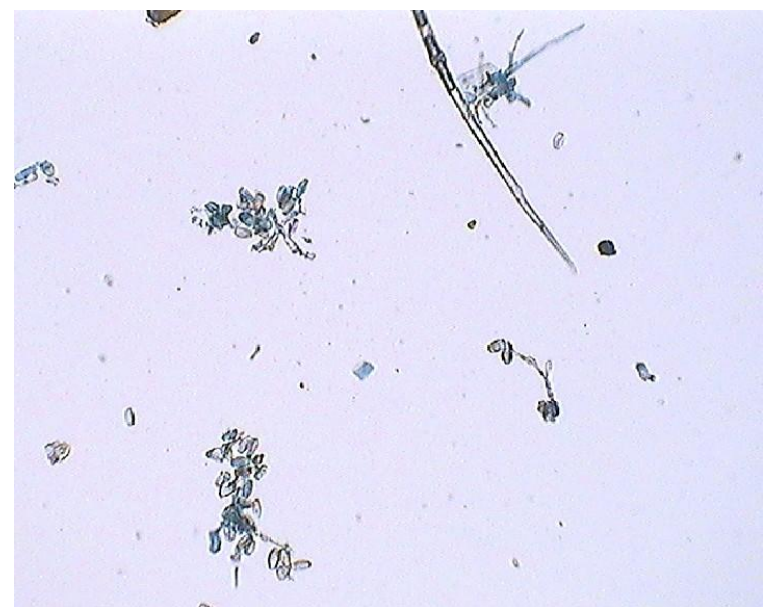

R-10-46-2A X R-393

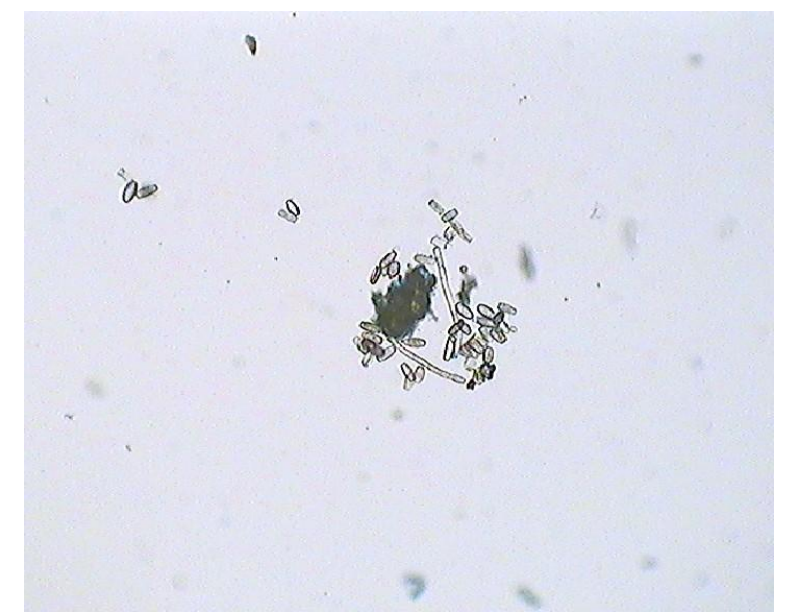

R-10-46-2A X R2F01120

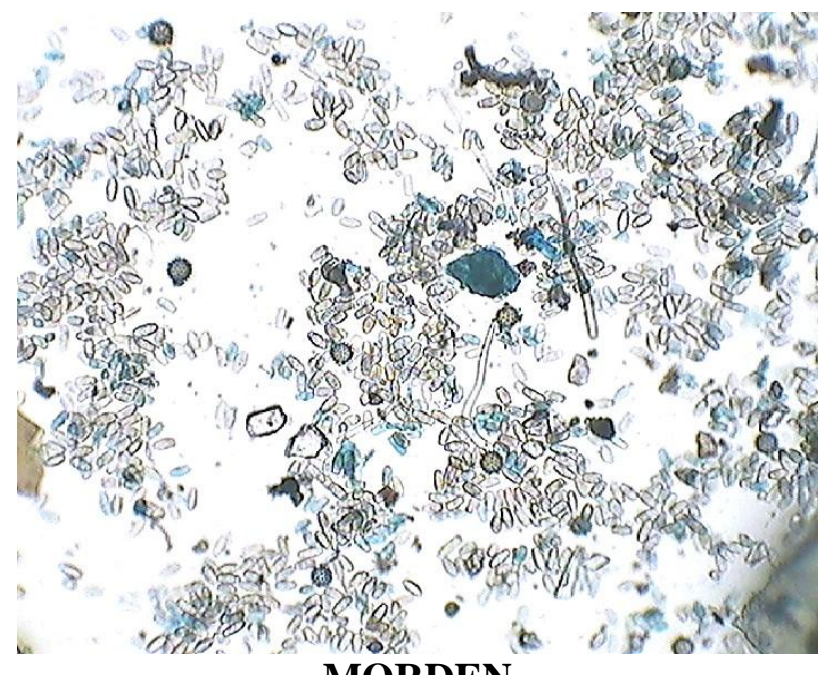

MORDEN

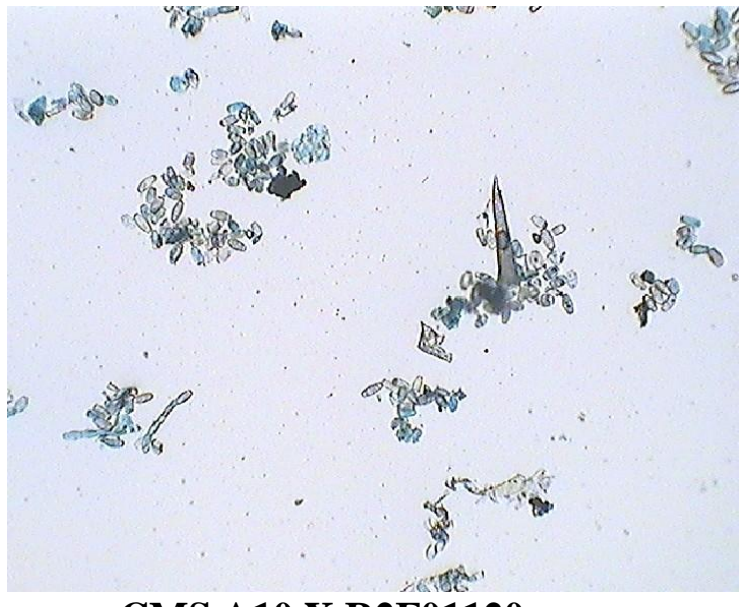

CMS A10 X R2F01120

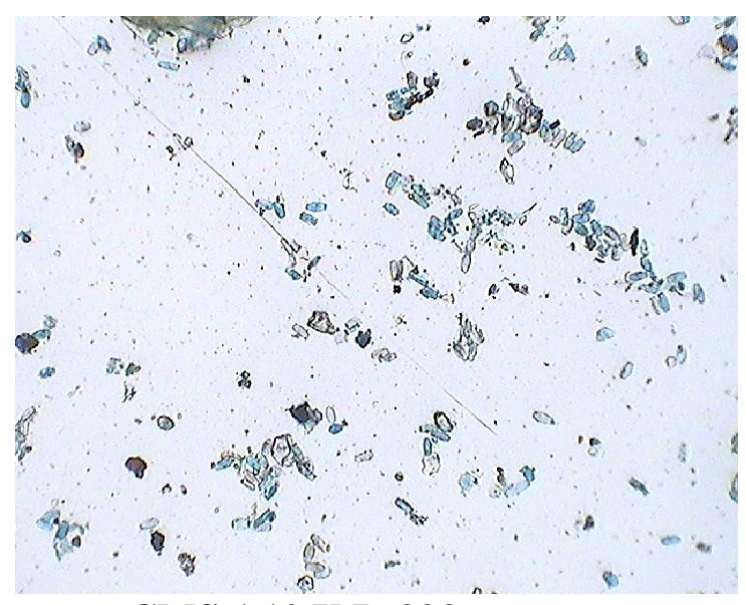

CMS A10 X R-393 
Fig.3 Average per cent disease severity in moderate resistant, susceptible and highly susceptible sunflower genotypes

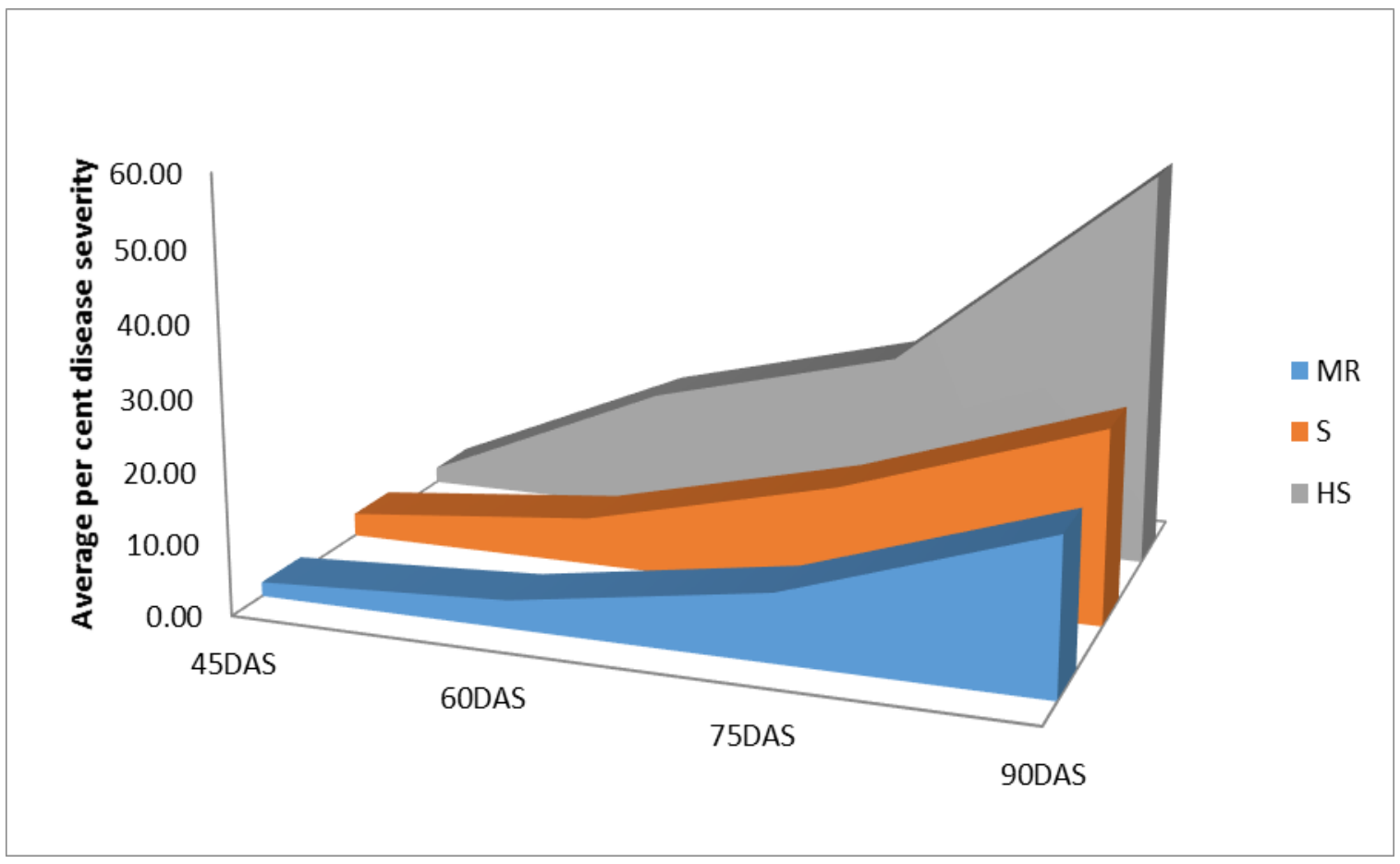

Fig.4 Differences in powdery mildew infection in moderate resistant, susceptible and highly susceptible sunflower genotypes

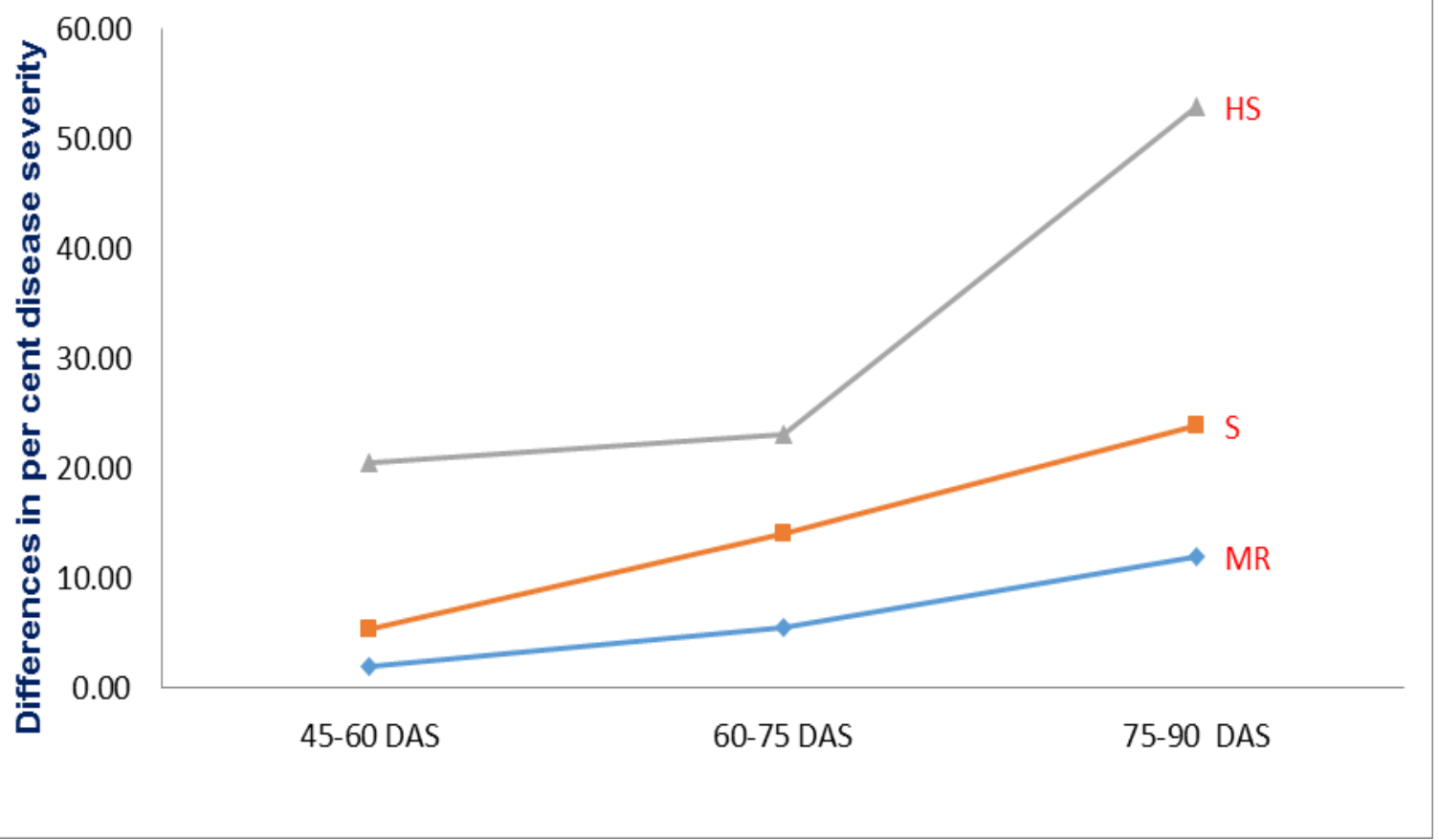


The genotypes viz., CMS A10 x R-GM-49, CMS A10 x 83-Br and CMS A10 x R-GM-39 having low apparent rate of infection actually recorded high disease infection at their early growth stage however infection rate was low. The genotypes CMS A10 x R-393, CMS A10 $\mathrm{x}$ R-GM-41 and RSFH-130 having high apparent rate of infection registered very low level of disease infection at their early crop growth stage, however once the disease infection occurs in these genotypes spread of the disease is fast. These results indicate the low apparent rate of infection does not indicate the resistant level of the genotype.

The calculated ' $r$ ' values varied and at times they did not remain consistent for given genotype and also did not show a particular trend in general.

This observation is in agreement with that of Wilcoxson et al., (1975) and Nargund (1989) who pointed out that ' $r$ ' values are not useful criteria for selecting the genotype. However, it can be used in studying the disease development in different genetic background.

The microscopic observation of the fungus was carried out on selected moderate resistant genotypes which scored least per cent disease severity at 90 days after sowing (Fig. 3).

For microscopic examination of pathogen, the infected top leaves were scraped gently to dislodge the conidia, then these conidia were stained with lacto phenol blue and observed under motic image capturing microscope at 10X (Figs. 1 and 2).

The number of conidia spores was counted in ten different microscopic fields for four selected moderate resistant genotypes, one susceptible and highly susceptible check Morden. The average number of conidia per microscopic field were analysed using DMRT (0.05). The moderate resistant genotypes $\mathrm{R}$ -
10-46-2A x R2F01120 (27.4) and R-10-462A x R-393 (34.6) recorded least number of conidia per microscopic field. The DMRT analysis categorised the average conidial count into 5 classes, indicating significant differences between 6 genotypes (Table 4). The other two moderate resistant hybrids CMS A10 x R-393 and CMS A10 x $\mathrm{R} 2 \mathrm{~F} 01120$ (B) recorded slightly higher number of conidial spores (152.5 and 102.9, respectively).

However, these conidial spores were significantly lower than susceptible hybrid CMS 850A x R-GM-69 (193.5) and highly susceptible check Morden (386). We could also observe significant differences for number of conidial spores in susceptible hybrid CMS 850A x R-GM-69 as compared to highly susceptible check Morden. These microscopic observations are in line with Reddy et al., (2013) they also reported less conidial spores and hyphal growth in resistant and moderately resistant sunflower genotypes compared to susceptible check (Fig. 4).

In conclusion, the present study clearly indicates that it is possible to synthesize hybrids with reasonable degree of tolerance by involving moderate tolerant genotype as one of the parent.

Further, resistance to powdery mildew is reported to exhibit differential reaction in different environmental conditions (Saliman, 1982) and several studies have identified resistance in wild species of sunflower. However, transferring resistant genes from wild species to cultivated species requires special techniques like ovule/embryo culture and moreover resistant genes come with linkage drag. Hence, in the absence of high level of resistance to powdery mildew, genes responsible for partial resistance are potentially useful for development of cultivars with durable resistance. 


\section{References}

Anonymous, 2016, Annual Group Meeting on Sunflower, Director's report, ICAR-Indian Institute of Oilseeds Research.

Dedic, B., Sreten, T., Jovanka, A., Dragana, M., Jelena, M., Sonja, T. and Vladimir, M., 2012, Screening perennial Helianthus species for powdery mildew. In: Proceedings of the 18th International Sunflower Conference, Argentina.

Goswami, B. K. and Dasgupta, M. A., 1981, Leaf blight, powdery mildew and charcoal rot diseases of sunflower from west Bengal. Indian Phytopath, 34(1): 14-16.

Gullino, M. L. and Kuijpers, L. M., 1994, Social and political implications of managing plant diseases with restricted fungicides in Europe. Annu. Rev. Phytopathol, 32:559579.

Hiremath, R.V., 1976, Studies on disease of sesamum. Incidence of powdery mildew on some varieties. J. oilseeds, 6(3): 18.

Karunanithi, K. and Dinakaran, D., 1996, Reaction of sesamum entries to Oidium acanthospermi. Madras Agric. J., 83(8): 541-542.

Mayee, C. D. and Datar, V. V., 1986, Phytopathometry. Marathwada Agric. Univ. Tech. Bull., 1: 46.

Nargund, V.B., 1989, Epidemiology and control of leaf rust of wheat caused by Puccinia recondita f.sp. tritici Rob. Ex. Desm. Ph.D. Thesis, Univ. Agric. Sci. Dharwad, Karnataka, India. p. 337.

Patel, M. K., Kamat, M. N. and Bhide, V. P., 1949, Fungi of Bombay. Indian Phytopath, 02: 142 .

Prasada, R., Jain, J. P. and Bhatnagar, M. K., 1968, Sphaerotheca fuliginea (Schlecht) Pollaci on sunflower (Helianthus annuus L.), a new record for Rajasthan, India.
Indian Phytopath, 21: 449-451.

Reddy, K.P., Rao, S.C., Kirti, P.B. and Sujatha, M., 2013, Development of a scoring scale for powdery mildew (Golovinomyces cichoracearum (DC.) V.P. Heluta) disease and identification of resistance sources in cultivated and wild sunflowers. Euphytica, 190:385-399.

Saliman, M., Yang, S.M. and Wilson, L., 1982, Reaction of Helianthus species to Erysiphe cichoracearum. Plant Dis., 66:572-573.

Seiler, G. J., 2008, Utilization of wild Helianthus species in breeding for disease resistance. In: Proceedings of the $17^{\text {th }}$ International Sunflower Conference, Cordoba, Spain, pp.709-713

Shadakshari, Y. G., Virupakshappa, K. and Siddaramaiah, A. L., 1989, Reactions of sesamum genotypes against powdery mildew and bacterial leaf spot. Current Res., 18(1): 4-5.

Singh, U. P., Singh, H. B. and Chauhan, V. B., 1984, Effect of some plant extracts and an oil on inoculum density of different nodal leaves of pea (Pisum sativum). $Z$. Plfanzenschutz, 91: 20-26.

Suresh, M., Narayanan, A., Ramasamy, R., Vaidyanathan, P. and Sree Rangaswamy, S. R., 1991, Sources of powdery mildew resistance in sesame. Sesame and Safflower newsletter, 6: 38-40.

Van der Plank, J.E., 1963, Plant Disease Epidemics and Control. Academic Press, New York, p. 349.

Wheeler, B.J. 1969. An introduction to plant disease, John Willey and Sons, Limited, London. P. 374.

Wilcoxson, R.D., Skovmand, B. and Atif, A.H., 1975, Evaluation of wheat cultivars for stability to retard development of stem rust. Ann. Appl. Bio., 80: 275-281.

\section{How to cite this article:}

Suresha, P.G., Vikas V. Kulkarni, S.M. Supriya, and Govindappa, M.R. 2017. Evaluation of Experimental Hybrids for Powdery Mildew Tolerance in Sunflower (Helianthus annuus L.). Int.J.Curr.Microbiol.App.Sci. 6(7): 2716-2728. doi: https://doi.org/10.20546/ijcmas.2017.607.381 